\title{
Differential impact of chronic stress along the hippocampal dorsal-ventral axis
}

\author{
V. Pinto $\cdot$ J. C. Costa $\cdot$ P. Morgado $\cdot$ \\ C. Mota $\cdot$ A. Miranda $\cdot$ F. V. Bravo $\cdot$ \\ T. G. Oliveira $\cdot$ J. J. Cerqueira $\cdot$ N. Sousa
}

Received: 26 June 2013 / Accepted: 23 January 2014

(C) Springer-Verlag Berlin Heidelberg 2014

\begin{abstract}
Stress impacts differently in distinct brain regions. However, so far few studies have focused on the differential responses triggered by stressful stimuli on the intrinsic functional heterogeneity of the hippocampal axis. In this study, we assessed the functional and structural alterations caused by exposure to a chronic unpredictable stress (CUS) paradigm on the dorsal-ventral axis of the hippocampus. The morphological analysis demonstrated that CUS had opposite outcomes in the structure of the dorsal (DH) and ventral hippocampus ( $\mathrm{VH})$ : whereas in the $\mathrm{DH}$, stress triggered a volumetric reduction as a result of atrophy of CA3 and CA1 apical dendrites, in the VH there was an increase in hippocampal volume concurrent with the increase of CA3 apical dendrites. In parallel, electrophysiological data revealed that stress led to a decrease in VH LTD. In summary, the present work showed that stress impacts differently on the structure and function of the $\mathrm{DH}$ and $\mathrm{VH}$ which contributes to better understand the overall spectrum of the central effects of stress.
\end{abstract}

Keywords Chronic stress - Hippocampal axis - LTP . LTD · Morphology

V. Pinto - J. C. Costa - P. Morgado - C. Mota - A. Miranda · F. V. Bravo - T. G. Oliveira - J. J. Cerqueira · N. Sousa $(\square)$ Life and Health Sciences Research Institute (ICVS), School of Health Sciences, University of Minho, Braga, Portugal e-mail: njcsousa@ecsaude.uminho.pt

V. Pinto - J. C. Costa P. Morgado - C. Mota $\cdot$ A. Miranda . F. V. Bravo - T. G. Oliveira - J. J. Cerqueira · N. Sousa ICVS/3B's-PT Government Associate Laboratory,

Braga/Guimarães, Portugal

\section{Introduction}

The stress response, largely mediated by the release of corticosteroids, is primarily an adaptive response. However, in the event that adequate adaptive mechanisms cannot be recruited, or when the intensity or duration of stressors exceeds a certain individual specific threshold, activation of the stress response is deleterious (reviewed by Sousa and Almeida 2012; Godsil et al. 2013). Several studies from different laboratories have, in fact, repeatedly demonstrated a stress-induced hippocampal dendritic atrophy (reviewed by McEwen 2012; Sousa and Almeida 2012), in parallel with functional deficits in hippocampal-dependent functions (Pavlides and McEwen 1999; Krugers et al. 2005; Cerqueira et al. 2007; reviewed by Segal et al. 2010).

The hippocampus serves a pivotal role in cognitive and emotional processes (reviewed by Fanselow and Dong 2010), as well as in the regulation of the HPA axis (reviewed by Sousa et al. 2008). Yet, there is solid evidence for a functional dissociation in the hippocampus: while the dorsal hippocampus (DH) is predominantly dedicated to cognitive processing, its ventral $(\mathrm{VH})$ counterpart is more implicated in emotional processing (McHugh et al. 2011; reviewed by Fanselow and Dong 2010). Importantly, the hippocampus is highly endowed with corticosteroid receptors (CRs) (reviewed by Sousa et al. 2008). Recent evidences also point to the different distribution of CRs along the hippocampal axis. While the DH has a higher prevalence of the lowaffinity glucocorticoid receptor (GR), the high-affinity mineralocorticoid receptor (MR) is more abundant in the $\mathrm{VH}$ (reviewed by Segal et al. 2010). Even though both receptors seem to modulate hippocampal networks, this modulation is biphasic. The MR is thought to be important for maintaining the excitability and stability of networks, prevailing as a positive modulator, whereas the GR seems to suppress or 
normalize network activity, mainly acting as a network suppressor (reviewed by Sousa et al. 2008; McEwen 2012). Therefore, activation of GRs is more effective in the DH than in the $\mathrm{VH}$, the opposite being true for the MRs. These differences were shown to underlie opposite responses of the $\mathrm{DH}$ and VH to acute stressful stimuli (reviewed by Segal et al. 2010). Curiously, stress-induced time-course evolution of corticosteroids in the $\mathrm{DH}$ and $\mathrm{VH}$ also follows a different pattern, as there is a functional switch from the $\mathrm{DH}$ to the $\mathrm{VH}$ after a single acute stressor (Dorey et al. 2012).

However, most of the previous studies were focused either on the $\mathrm{DH}$ or on acute stress paradigms (reviewed by McEwen 2012; Sousa and Almeida 2012). Therefore, it seems critical to study the impact of chronic unpredictable stress (CUS), a stress paradigm closer to real-life situations, along the entire hippocampal axis. To this aim, we used a CUS paradigm previously validated in our group (Cerqueira et al. 2007) and performed a stereological assessment of the volumes of discrete hippocampal divisions and a 3D morphometric analysis of dendritic arborizations of hippocampal neurons. Additionally, we measured the ability to induce long-term potentiation (LTP) and depression (LTD), two cellular models of learning and memory (reviewed by Bear and Abraham 1996; Bliss and Collingridge 1993), in DH and VH of control (CON) and CUS animals.

\section{Materials and methods}

Animals and treatments

Experiments were conducted in accordance with local regulations (European Union Directive 2010/63/EU) and National Institutes of Health guidelines on animal care and experimentation.

Two-month-old male Wistar rats $(n=62)$ (Charles River Laboratories, Barcelona, Spain) were used and divided into two subgroups. A subgroup of animals $(n=33)$ was submitted to 4 weeks CUS (Cerqueira et al. 2007). Briefly, animals were exposed once daily to a stressor $(1 \mathrm{~h} /$ day $)$ of one of several aversive stimuli [cold water $\left(18{ }^{\circ} \mathrm{C}\right)$, vibration, restraint, overcrowding, exposure to a hot air stream]; the stressors were presented in random order for the duration of the experiment. Another subgroup $(n=29)$ was handled daily and served as control.

Body weights were monitored weekly for a subset of animals as confirmation of treatment efficacy.

After exposure to 4 weeks CUS and handling, a subset of animals (CON, $n=10$; CUS, $n=10)$ was behaviorally tested [first in the elevated plus maze (EPM) and then in the Morris water maze (MWM)] and subjected to morphological analysis. Another subset (CON, $n=14$; CUS, $n=16$ ) was used for electrophysiological recordings. The recordings took place in a period of up to 2 weeks, in which the animals were kept under stress exposure. Another subset of animals (CON, $n=5$; CUS, $n=7$ ) was directly subjected to morphological analysis after the CUS paradigm. All behavioral manipulations of the animals, including the CUS paradigm, were done during the daytime.

Behavioral procedures

\section{Elevated plus maze}

Experiments were performed as previously described (Pêgo et al. 2008). Briefly, rats were placed in the center of the maze facing a closed arm and were allowed 5 min of free exploration. The percentage of time spent in the open arms was used to assess anxiety.

\section{Morris water maze}

The reference memory task was performed as previously described (Cerqueira et al. 2007). Briefly, the test consisted of 4 days of acquisition (4 trials/day). During the 4 days of trials, the position of the hidden platform was kept constant, but animals were placed facing the wall of the maze at different starting point positions. The time spent to reach the platform (escape latency) was recorded in consecutive trials.

Histological procedures

\section{Volumetric estimations}

Experiments were performed according to the protocol described by Cerqueira et al. (2007). Briefly, animals (CON, $n=4$; CUS, $n=5$ ) were perfused transcardially with fixative (4\% paraformaldehyde), under deep pentobarbital anesthesia, and brains were removed for volume estimation. Every tenth section $(30 \mu \mathrm{m})$ was analyzed. Individual volumes of the dentate gyrus (DG), CA3 and CA1 were added to give the final volume of the hippocampal formation (HF). The shrinkage factor did not differ significantly among experimental groups.

\section{$3 D$ dendritic analysis}

Three-dimensional reconstructions of DG, CA3 and CA1 neurons were performed as previously described (Pêgo et al. 2008). Briefly, animals (CON, $n=11$; CUS, $n=12$ ) were transcardially perfused with $0.9 \%$ saline under deep pentobarbital anesthesia and brains were removed and immersed in Golgi-Cox solution. Coronal sections (200 $\mu \mathrm{m}$ thick) were collected for neuron reconstruction. For each animal, five neurons per sub-region were analyzed. 
Electrophysiological analysis

\section{Slice preparation}

Animals were anesthetized with halothane and decapitated. The brain was quickly removed in ice-cold sucrose-based artificial cerebrospinal fluid solution containing the following (in $\mathrm{mM}$ ): $2.5 \mathrm{KCl}, 7 \mathrm{MgCl}_{2}, 1.25 \mathrm{NaH}_{2} \mathrm{PO}_{4}, 110$ sucrose, $26 \mathrm{NaHCO}_{3}, 10$ glucose, bubbled with carbogen gas $(95 \%$ $\left.\mathrm{O}_{2}, 5 \% \mathrm{CO}_{2}\right)$. Axial slices $(300 \mu \mathrm{m})$ taken from the two extremes $(2 \mathrm{~mm})$ of the hippocampus were cut using a tissue slicer (Leica VT $1,200 \mathrm{~s})$. Recordings $\left(31^{\circ} \mathrm{C}\right)$ were performed in a submerged chamber with standard artificial cerebrospinal fluid containing (in $\mathrm{mM}$ ): $124 \mathrm{NaCl}, 2.5 \mathrm{KCl}, 1$ $\mathrm{MgSO}_{4}, 2 \mathrm{CaCl}_{2}, 1.25 \mathrm{NaH}_{2} \mathrm{PO}_{4}, 26 \mathrm{NaHCO}_{3}, 10$ glucose, bubbled with carbogen gas $\left(95 \% \mathrm{O}_{2}, 5 \% \mathrm{CO} 2\right)$.

\section{Recordings}

Extracellular recordings were made with a Multiclamp 700B amplifier (Axon Instruments) and borosilicate glass pipettes (3-5 M $\Omega$ ) filled with saline $(0.75 \mathrm{M} \mathrm{NaCl})$. Signals were lowpass filtered at $3 \mathrm{kHz}$ and sampled at $10 \mathrm{kHz}$. Stimulation was performed using a bipolar tungsten electrode. Both recording and stimulating electrodes were placed in the middle of the stratum radiatum of CA1. Slices unable to produce a field excitatory postsynaptic potential (fEPSP) of $1 \mathrm{mV}$ were discarded. Stimulus strength was adjusted to $30-50 \%$ of the maximum fEPSP slope. In some animals, the paired pulse (PP) ratio was also assessed before LTP induction by giving two close stimuli of varying interpulse interval $(25,50,100$ and $300 \mathrm{~ms}$ ). The ratio was calculated by dividing the slope of fEPSP 2 by fEPSP 1 . Baselines were acquired at $0.03 \mathrm{~Hz}$. LTP was elicited by delivering $3 \theta$-burst stimuli ( $\theta$-burst: $100 \mathrm{~Hz}$ burst of four pulses repeated at $5 \mathrm{~Hz}$ with each tetanus including 10-burst trains) separated by $15 \mathrm{~s}$ intervals. LTD was induced by applying a low-frequency stimulation protocol consisting of $15 \mathrm{~min}$ of $1 \mathrm{~Hz}$ stimulation. Final slopes were calculated off-line using the LTP software (Anderson and Collingridge 2007). All points of each individual curve were normalized to the average value of the last $10 \mathrm{~min}$ baseline. Averages of the slopes of the last 6 min recordings were used for comparison. For each animal, only $1 \mathrm{DH}$ slice and $1 \mathrm{VH}$ slice were used for recording.

\section{Statistical analysis}

Statistical analysis was conducted using IBM SPSS Statistics. Results are expressed as group mean \pm SEM. Data points were considered outliers if they were three standard deviations apart from the group mean. Normality of sampling distribution within groups was confirmed using the Kolmogorov-Smirnov and Shapiro-Wilk tests. The MWM and PP curves were analyzed using ANOVA. For experiments with two groups, the Student's $t$ test was used. Differences were considered to be significant if $p<0.05$.

\section{Results}

Chronic stress affects hippocampal-dependent behaviors and biometric parameters

Analysis of the times spent in the open arms of the EPM (Fig. 1b) revealed that CUS rats were more anxious than CON and spent less time in the open arms ( $t$ test; CON, $15.76 \pm 1.87 \%$ vs. CUS, $8.64 \pm 1.33 \% ; t(18)=3.10$; $p=0.0062$ ).

The analysis of the latency curves in the spatial reference memory task (Fig. 1a) revealed a significant effect of stress (ANOVA; $\left.F_{(1,16)}=12.319 ; p=0.003\right)$. CUS rats showed impaired learning ability, taking more time to find the hidden platform in the second ( $t$ test; CUS, $56.88 \pm 4.20 \mathrm{~s}$ vs. $\mathrm{CON}, 29.50 \pm 4.16 \mathrm{~s} ; t(16)=-4.57$; $p=0.0003$ ) day of the test.

The efficacy of the CUS paradigm was proven by its effect on body weight. In accordance with previous observations (Cerqueira et al. 2007), CUS decreased the percentage of body-weight gain in both CON $(n=14)$ and CUS $(n=16)$ animals $(t$ test; CON, $126.1 \pm 2.2 \%$ vs. CUS, $119.6 \pm 1.8 \% ; t(28)=2.32 ; p=0.0281)$.

Differential structural effects of chronic stress along the hippocampal dorsal-ventral axis

Analyses of the effects of CUS on volume alterations were done in five contiguous hippocampal divisions (A to E: A is the most dorsal and $\mathrm{E}$ the most ventral) (Fig. 2a) and revealed that, overall, the two poles of the hippocampus
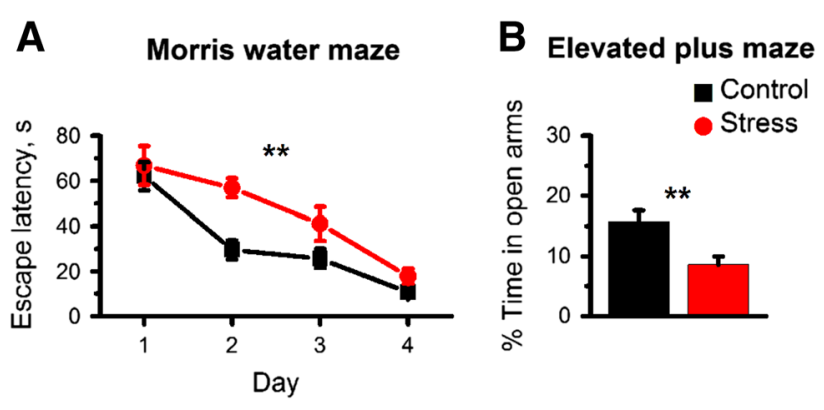

Fig. 1 CUS impairs cognition and increases anxiety-like behavior. a Learning curves in the MWM show CUS animals $(n=8)$ have impaired learning abilities as compared to CON animals $(n=10)$. b Percentage of time spent in the open arms of the EPM. CUS rats $(n=10)$ show increased anxiety-like behavior as compared to CON $(n=10) . * * p<0.01$ 

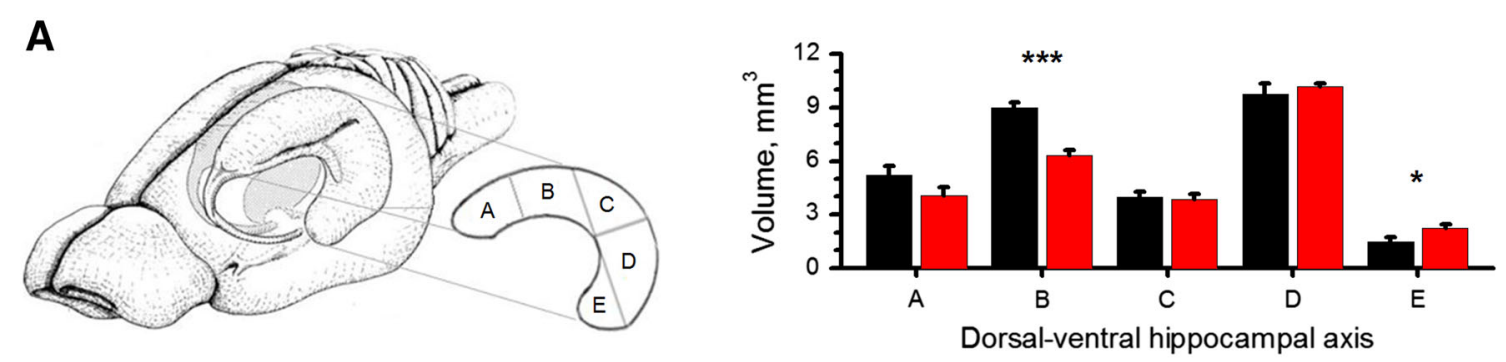

- Control

- Stress

Dorsal hippocampus Ventral hippocampus

B

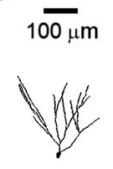

C

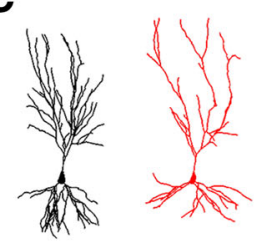

D
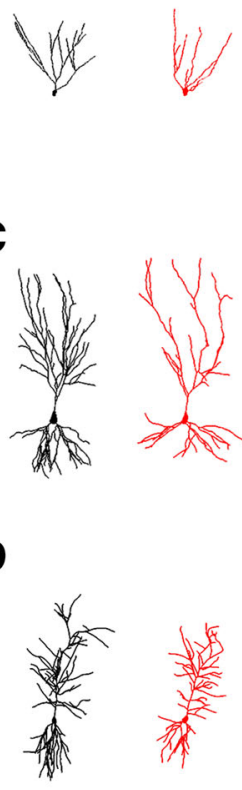

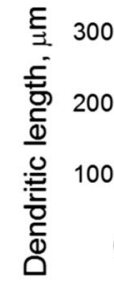
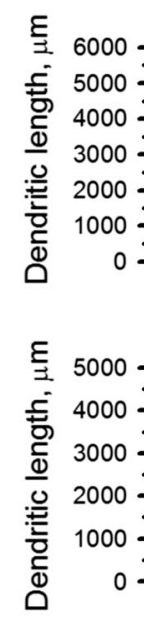

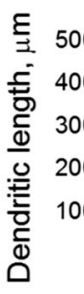

Fig. 2 The morphology of the hippocampal axis is differently affected by CUS. a Illustration shows five contiguous regions (ae) along the hippocampal axis. Differences between CON animals $(n=4)$ and CUS animals $(n=5)$ vary depending the position along the axis. b-d Analyses of dendritic lengths of granular (DG) and pyramidal cells (CA3, CA1) of the hippocampus. DH corresponds to

have opposite alterations in volume when challenged with CUS, with the dorsal pole presenting a volume reduction and the ventral pole a volume increase ( $t$ test; A: CON, $5.23 \pm 0.46 \mathrm{~mm}^{3}$ vs. CUS, $4.07 \pm 0.48 \mathrm{~mm}^{3} ; t(7)=1.70$; $p=0.1326 ; \quad$ B: $\mathrm{CON}, \quad 9.00 \pm 0.26 \mathrm{~mm}^{3}$ vs. CUS, $6.29 \pm 0.32 \mathrm{~mm}^{3} ; t(7)=6.32 ; p=0.0004 ; \mathrm{C}: \mathrm{CON}$, $3.99 \pm 0.29 \mathrm{~mm}^{3}$ vs. CUS, $3.84 \pm 0.33 \mathrm{~mm}^{3} ; t(7)=0.32$; $p=0.7560 ; \quad \mathrm{D}: \mathrm{CON}, \quad 9.76 \pm 0.60 \mathrm{~mm}^{3}$ vs. CUS, $10.17 \pm 0.17 \mathrm{~mm}^{3} ; t(7)=-0.74 ; p=0.4830 ; \mathrm{E}: \mathrm{CON}$, $1.49 \pm 0.28 \mathrm{~mm}^{3}$ vs. CUS, $2.26 \pm 0.18 \mathrm{~mm}^{3} ; \quad t(7)=$ $-2.42 ; p=0.0458$ ) (Fig. 2a).

DG
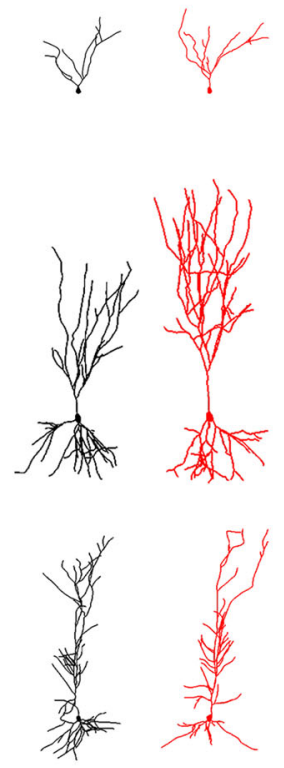

region $\mathrm{A}+\mathrm{B}$ and $\mathrm{VH}$ to region $\mathrm{D}+\mathrm{E}$. Measurements in $\mathrm{CON}$ animals (DG DH, $n=11$; DG VH, $n=9$; CA3 DH, $n=6$; CA3 VH, $n=8$; CA1 DH, $n=6$; CA1 VH, $n=6$ ) and CUS animals (DG DH, $n=12$; DG VH, $n=10$; CA3 DH, $n=7$; CA3 VH, $n=9$; CA1 $\mathrm{DH}, n=6$; CA1 $\mathrm{VH}, n=6$ ) show opposite variations between the two hippocampi. $* p<0.05 ; * * p<0.01 ; * * * p<0.001$

A detailed analysis on the dendritic trees of granule and pyramidal cells, along the dorsal-ventral axis (regions $\mathrm{A}+\mathrm{B}$ related with the $\mathrm{DH}$ and regions $\mathrm{D}+\mathrm{E}$ related with the $\mathrm{VH}$, respectively; see illustration in Fig. 2a) of the HF, comprising DG, CA3 and CA1, revealed CUS also had different outcomes in the average lengths of dendrites.

CUS caused a non-significant decrease in the average length of the dendrites of DH granule cells ( $t$ test; CON, $2,020.7 \pm 147.2 \mu \mathrm{m} \quad$ vs. CUS, $1,729.9 \pm 64.4 \mu \mathrm{m}$; $t(13.74)=-1.81 ; p=0.0920)$ and no net alteration in the dendrites of $\mathrm{VH}$ granule cells ( $t$ test; CON, 2,034.9 
$\pm 193.6 \mu \mathrm{m} \quad$ vs. CUS, $2,117.7 \pm 189.8 \mu \mathrm{m} ; \quad t(17)=$ 0.0305; $p=0.7641$ ) (Fig. 2b).

The apical dendrites of $\mathrm{DH}$ and $\mathrm{VH}$ CA3 pyramidal neurons were distinctly affected by CUS. While CUS caused a shortening of the apical dendrites of DH pyramidal neurons ( $t$ test; CON, 3,614.7 $\pm 145.1 \mu \mathrm{m}$ vs. CUS, $2,880.8 \pm 178.8 \mu \mathrm{m} ; \quad t(11)=-3.11 ; \quad p=0.0099)$, the opposite occurred in the $\mathrm{VH}$ which had an increase in the average dendritic length of pyramidal neurons ( $t$ test; CON, $4,682.7 \pm 164.8 \mu \mathrm{m}$ vs. CUS, 5,645.4 $\pm 285.0 \mu \mathrm{m}$; $t(12.63)=-2.92 ; p=0.0122)$. Basal dendrites of CA3 pyramidal neurons were not significantly affected by CUS in either region ( $t$ test; DH: CON, 2,442.9 $\pm 159.5 \mu \mathrm{m}$ vs. CUS, $2,091.8 \pm 163.0 \mu \mathrm{m} ; t(11)=-1.53 ; p=0.1550$; VH: CON, $3,378.6 \pm 161.0 \mu \mathrm{m}$ vs. CUS, 3,081.4 \pm $149.4 \mu \mathrm{m} ; t(15)=-1.35 ; p=0.1958$ ) (Fig. 2c).

CUS also had dissimilar outcomes in CA1 pyramidal neurons of $\mathrm{DH}$ and $\mathrm{VH}$. While there were no significant alterations in the apical dendrites of the $\mathrm{VH}$ ( $t$ test; CON, 4,103.1 $\pm 307.7 \mu \mathrm{m}$ vs. CUS, 4,232.4 $\pm 273.4 \mu \mathrm{m}$; $t(10)=0.31 ; p=0.7598)$, apical dendrites of DH pyramidal neurons showed a substantial shrinkage ( $t$ test; CON, 4,425.8 $\pm 211.8 \mu \mathrm{m}$ vs. CUS, 3,084.6 $\pm 148.2 \mu \mathrm{m}$; $t(10)=-5.19 ; p=0.0004)$. Again, there were no significant alterations in the dendritic lengths of the basal dendrites of CA1 pyramidal neurons in both the DH and VH ( $t$ test; DH: CON, $2,176.0 \pm 125.7 \mu \mathrm{m}$ vs. CUS, $1,852.5 \pm 197.9 \mu \mathrm{m} ; \quad t(10)=-1.38 ; \quad p=0.1977 ; \quad \mathrm{VH}:$ CON, $1,767.9 \pm 124.2 \mu \mathrm{m}$ vs. CUS, $1,846.8 \pm 133.7 \mu \mathrm{m}$; $t(10)=0.433 ; p=0.6744$ ) (Fig. 2d).

Chronic stress affects the induction of LTD in the ventral hippocampus

The effects of CUS in DH and $\mathrm{VH}$ synaptic activity was assessed in the CA1 region of the HF. As already mentioned, this region presented important morphological alterations when challenged by CUS, being also influenced by the effects of CUS in CA3, its major source of afferent inputs.

In agreement with previous findings (Maggio and Segal 2007), the DH presented a higher LTP than the VH under basal conditions ( $t$ test; $\mathrm{DH}, 1.24 \pm 0.06$ vs. $\mathrm{VH}$, $1.04 \pm 0.03 ; t(8)=3.03 ; p=0.0162$ ) (Fig. 3a, b). This difference was also apparent after stress exposure, which did not alter LTP in either region ( $t$ test; DH: CON, $1.24 \pm 0.06$ vs. CUS, $1.25 \pm 0.04 ; \quad t(11)=-0.19$; $p=0.8493 ; \quad \mathrm{VH}: \quad \mathrm{CON}, \quad 1.04 \pm 0.03 \quad$ vs. CUS, $1.13 \pm 0.08 ; t(10)=-0.94 ; p=0.3710)$ (Fig. 3a, b).

Although, as we have shown, LTP in the VH is of far lesser magnitude than in the $\mathrm{DH}$, this region is known to produce LTDs of similar magnitudes as the ones expressed in the DH (Maggio and Segal 2007). In accordance, our results show that the $\mathrm{DH}$ and $\mathrm{VH}$ of $\mathrm{CON}$ animals have similar LTD ( $t$ test; DH, $0.84 \pm 0.04$ vs. VH, $0.86 \pm 0.02$; $t(9)=-0.46 ; p=0.6561$ ) (Fig. 3c, d). Interestingly, the effect of CUS on LTD expression resulted in an increased activity of the $\mathrm{VH}$ as compared to its dorsal counterpart. This was due to the fact that CUS significantly decreased LTD in the former region, whereas no changes were observed in the DH ( $t$ test; DH: CON, $0.84 \pm 0.04$ vs. CUS, $0.91 \pm 0.04 ; t(8)=-1.08 ; p=0.3134 ; \mathrm{VH}$ : CON, $0.86 \pm 0.02$ vs. CUS, $0.95 \pm 0.01 ; t(10)=-3.33 ; p=$ 0.0076) (Fig. 3c, d).

To assess if the effects of CUS in synaptic plasticity could also be explained by presynaptic changes, we applied a PP protocol to $\mathrm{DH}$ and $\mathrm{VH}$ slices (Fig. 3e, f). ANOVA showed the PP curves of $\mathrm{CON} \mathrm{DH}$ and $\mathrm{VH}$ to be significantly different between them (ANOVA; $F_{(1,8)}=8.93$; $p=0.0170$ ), once again reinforcing the intrinsic functional difference between these two regions. However, CUS had no significant change in the $\mathrm{PP}$ ratios of both the $\mathrm{DH}$ (ANOVA; $\left.F_{(1,10)}=0.169 ; p=0.6900\right)$ and VH (ANOVA; $\left.F_{(1,9)}=0.172 ; p=0.6880\right)$ (Fig. 3e, f).

\section{Discussion}

In this study we confirmed that CUS produced cognitive deficits and increased anxiety (Fig. 1a, b); however, for the first time, we showed that CUS triggered distinct structural and functional alterations in the dorsal and ventral poles of the hippocampus. On one hand, CUS reduced DH volume (Fig. 2a) and shortened CA3 and CA1 apical dendrites (Fig. 2c, d). On the other hand, the VH presented a volumetric enlargement concomitant with an increase in the length of CA3 apical dendrites (Fig. 2a, c). We could not exclude, however, that alterations in astrocytic number and axonal myelination could add up to the observed changes in neuronal branching contributing to changes in volume across the dorsal and ventral hippocampi. Nevertheless, these morphological dissimilarities were reinforced by assessments of synaptic activity as the CA1 region of the VH presented reduced LTD levels (Fig. 3d), suggesting a CUS-dependent ability to boost its activity not observed in its dorsal counterpart.

Chronic stress is known to cause behavioral changes in humans and rodents manifested as depressive-like symptoms, hyperanxious states, and learning and memory deficits (reviewed by Sousa and Almeida 2012; Godsil et al. 2013). These changes are often concomitant with structural alterations in several brain regions including hippocampus and prefrontal cortex (reviewed by McEwen 2012; Sousa and Almeida 2012; Godsil et al. 2013) atrophy, and dorsolateral striatum (Dias-Ferreira et al. 2009) and extended amygdala (Pêgo et al. 2008; reviewed by McEwen 2012; 
A

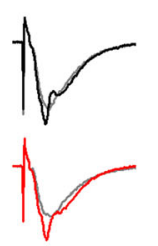

C

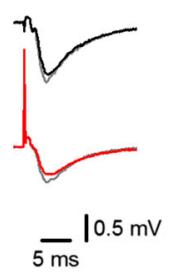

- Control

Stress
Dorsal hippocampus
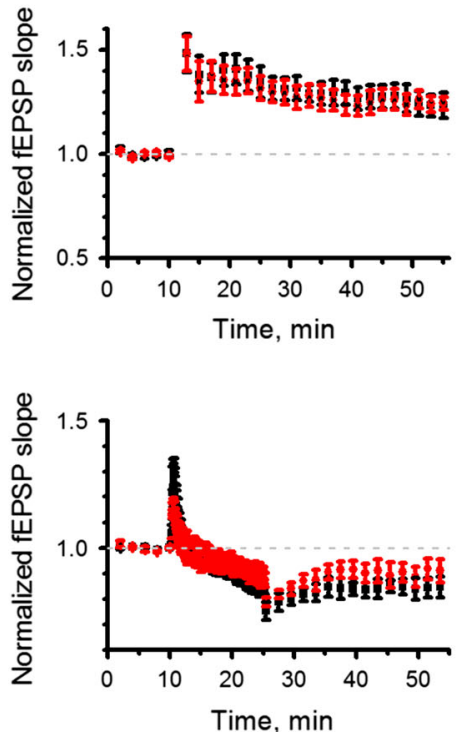

E

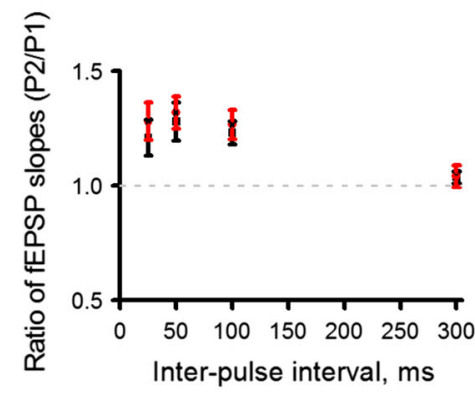

Fig. 3 CUS reduces LTD in the VH. a, b LTP was independently measured in acute slices of DH (CON, $n=5$; CUS, $n=8)$ and VH (CON, $n=5$; CUS, $n=7$ ), respectively. $\mathbf{c}, \mathbf{d}$ As for LTP, LTD was assessed in acute slices of the DH (CON, $n=5$; CUS, $n=5$ ) and VH (CON, $n=6$; CUS, $n=6$ ) respectively. Representative traces on the

Sousa and Almeida 2012) hypertrophy. The present morphological results are largely in accordance with these previous studies as we show that the $\mathrm{DH}$, on which previous studies largely focused, displays a pronounced atrophy. However, for the first time, we show that the VH, intimately related to the extended amygdala, shows the opposite outcome.

The laminar organization of the hippocampal formation is not a simple repetition of functional and structurally homogeneous parallel circuits. Although the basic wiring diagram of each hippocampal strip is the unidirectional trisynaptic loop (DG-CA3-CA1) (reviewed by Witter and Amaral 2004), there is great heterogeneity along the dorsalventral hippocampal axis. In fact, these two poles present anatomical segregation of input and output connections, such that the DH primarily projects to the neocortex, while the VH primarily impinges on subcortical structures, including the amygdala and hypothalamus (Swanson et al. 1978; Naber and Witter 1998; reviewed by Moser and

B
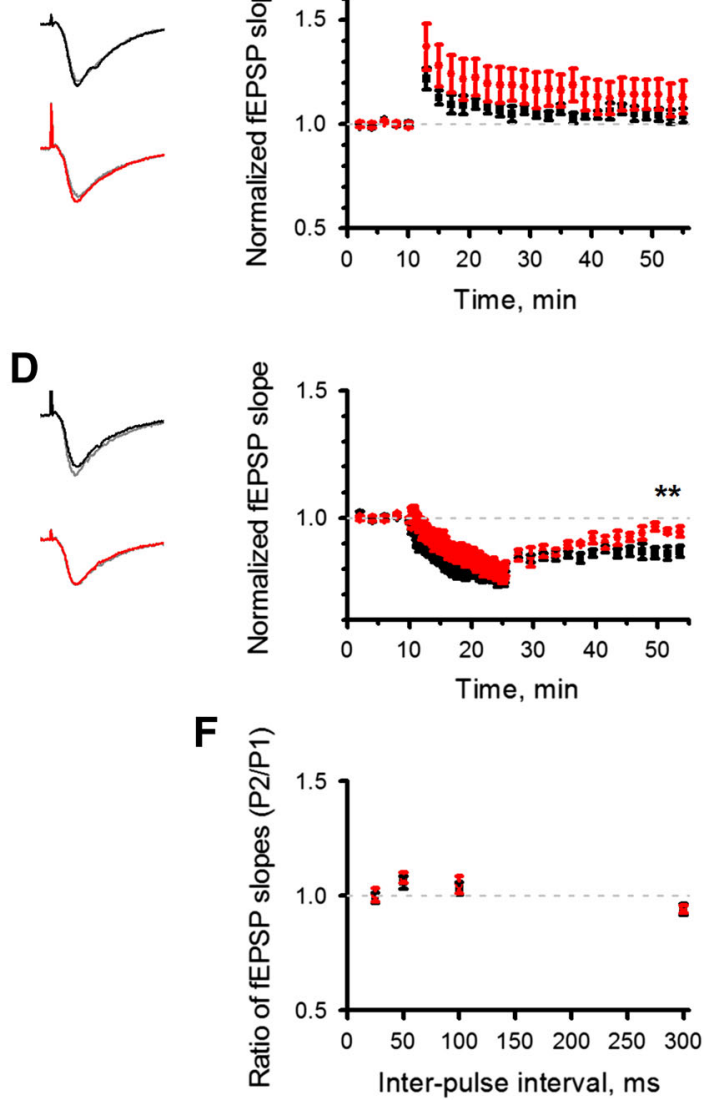

left are averages of four consecutive recordings (gray lines are baseline recordings, whereas colored lines were taken from the last 6 min recordings). e, $\mathbf{f}$ Paired pulse ratios for dorsal (CON, $n=5$; CUS, $n=7)$ and ventral (CON, $n=5$; CUS, $n=6$ ) acute hippocampal slices. $* * p<0.01$

Moser 1998; Fanselow and Dong 2010; Segal et al. 2010). Probably as a direct consequence, both hippocampi are implicated in different functions: the $\mathrm{DH}$ is involved in memory and cognitive processing, whereas the $\mathrm{VH}$ processes information related to the emotional and homeostatic states of the animal (McHugh et al. 2011; reviewed by Moser and Moser 1998; Fanselow and Dong 2010; Segal et al. 2010). In our study, we showed that, despite the obvious segregation of afferent and efferent connections, the $\mathrm{DH}$ and $\mathrm{VH}$ also show intrinsic differences in the mechanisms of information processing. This intrinsic difference was most obvious in the ability of the two hippocampi to express LTP. As previously reported by others (Papatheodoropoulos and Kostopoulos 2000a; Maruki et al. 2001; Colgin et al. 2004; Maggio and Segal 2007), the DH of CON animals expressed higher LTP as compared to the VH. Differences were also evident when we analyzed short-term plasticity in both regions. In this regard, the DH also presented higher levels of PP facilitation as compared to the $\mathrm{VH}$ 
(Papatheodoropoulos and Kostopoulos 2000b; Maruki et al. 2001). PP facilitation is directly related to the release probability of synaptic vesicles. If a synapse has a high release probability, as in the case of $\mathrm{VH} \mathrm{CA} 1$, it tends to depress the response in a second, very close stimulus due to a transient depletion of the release-ready pool of synaptic vesicles (reviewed by Zucker and Regehr 2002; Citri and Malenka 2008). As a result, CA1 synapses of the VH function as low-pass filters, since they will preferentially transmit low-frequency activity. This presynaptic property of the VH is thus finely tuned with its lower LTP inducibility. Further studies should be addressed to assess if these two properties of the $\mathrm{VH}$ are indeed interdependent. Besides lower synaptic plasticity, VH CA1 neurons also present lower excitability than that of the dorsal counterpart (reviewed by Segal et al. 2010). Nevertheless, it should be stressed that both DH and VH presented similar LTD levels in CON conditions (Maggio and Segal 2007), suggesting that the difference in plasticity did not reflect a lower modification threshold of the ventral synapse (Bear et al. 1987), but rather an intrinsic selective difference in plasticity-generating mechanisms.

This lower net activity of the VH suggests that its modifiability (i.e., metaplasticity) is higher than in the $\mathrm{DH}$ (Hessler et al. 1993; reviewed by Citri and Malenka 2008; Segal et al. 2010). Indeed, acute stress is able to significantly upregulate neuron excitability and synaptic activity in the VH (reviewed by Segal et al. 2010), and CUS significantly increases the power spectrum of the $\mathrm{VH}$ (Oliveira et al. 2013). Our present observations using a CUS model confirmed an increase in $\mathrm{VH}$ activity, due to a reduction in the ability to induce LTD (reviewed by Bear and Abraham 1996; Citri and Malenka 2008; Segal et al. 2010). It is important to note, however, that the results of our study are apparently partially in contradiction with studies of Hawley and Leasure (2012), and Hawley et al. (2012), showing that CUS animals selectively enhanced the activity of the DH, having a significantly better long-term spatial memory, while negatively impacted the VH. Besides the use of a shorter stress paradigm (Luine et al. 1996), the use of Long Evans rats may contribute to explain such discrepancies between studies, as this strain is known to have a higher adaptability (Ambrogi Lorenzini et al. 1987). It is thus critical to consider that comparisons between acute and chronic stress should be carefully approached as the longlasting effects of chronic stress might rely on more complex and diverse mechanisms. In fact, the impact of the stress response may be bi-modal with short exposure triggering adaptive beneficial effects and chronic exposure being detrimental (Sapolsky et al. 1985; Luine et al. 1994, 1996; Maggio and Segal 2007).

The morphological deficits we reported in the $\mathrm{DH}$ were apparently not accompanied by changes in synaptic plasticity.
This could be explained by recovery from acute changes in synaptic plasticity, during the progression of the chronic stress paradigm, but also by the mechanistic independence of both morphological and synaptic plasticity (Wang et al. 2007; Bastrikova et al. 2008). This points to the importance of performing longitudinal assessments when studying CUS paradigms. Curiously, a lifetime of stress, able to shape the course of brain aging (reviewed by Sotiropoulos et al. 2008), could account for the age-related deficits in context memory performance in older individuals. Aging was associated with volume loss in the anterior, but not the posterior hippocampus in a group of old-age human subjects. These changes were able to cause an age-dependent shift from anterior hippocampalrelated processes to lateral PFC-related processes (Rajah et al. 2010). Understanding the mechanisms of chronic stress may thus prove useful for healthy aging as well as for the development of new therapies for a variety of mental disorders.

Acknowledgments Pinto $\mathrm{V}$ and Mota $\mathrm{C}$ were supported by Fundação para a Ciência e Tecnologia (FCT) grants (SFRH/BPD/69132/ 2010; SFRH/BD/81881/2011, respectively). This work was supported by an FCT grant (PTDC/SAU-NSC/120590/2010). The authors declare no competing financial interests.

\section{References}

Ambrogi Lorenzini C, Bucherelli C, Giachetti A, Tassoni G (1987) Spontaneous and conditioned behavior of Wistar and Long Evans rats. Arch Ital Biol 125:155-170

Anderson WW, Collingridge GL (2007) Capabilities of the WinLTP data acquisition program extending beyond basic LTP experimental functions. J Neurosci Methods 162:346-356

Bastrikova N, Gardner GA, Reece JM, Jeromin A, Dudek SM (2008) Synapse elimination accompanies functional plasticity in hippocampal neurons. Proc Natl Acad Sci USA 105:3123-3127

Bear MF, Abraham WC (1996) Long-term depression in hippocampus. Annu Rev Neurosci 19:437-462

Bear MF, Cooper LN, Ebner FF (1987) A physiological basis for a theory of synapse modification. Science 237:42-48

Bliss TV, Collingridge GL (1993) A synaptic model of memory: long-term potentiation in the hippocampus. Nature 361:31-39

Cerqueira JJ, Mailliet F, Almeida OF, Jay TM, Sousa N (2007) The prefrontal cortex as a key target of the maladaptive response to stress. J Neurosci 27:2781-2787

Citri A, Malenka RC (2008) Synaptic plasticity: multiple forms, functions, and mechanisms. Neuropsychopharmacology 33:18-41

Colgin LL, Kubota D, Jia Y, Rex CS, Lynch G (2004) Long-term potentiation is impaired in rat hippocampal slices that produce spontaneous sharp waves. J Physiol 558:953-961

Dias-Ferreira E, Sousa JC, Melo I, Morgado P, Mesquita AR, Cerqueira JJ, Costa RM, Sousa N (2009) Chronic stress causes frontostriatal reorganization and affects decision-making. Science 325:621-625

Dorey R, Piérard C, Chauveau F, David V, Béracochéa D (2012) Stress-induced memory retrieval impairments: different timecourse involvement of corticosterone and glucocorticoid receptors in dorsal and ventral hippocampus. Neuropsychopharmacology 37:2870-2880

Fanselow MS, Dong HW (2010) Are the dorsal and ventral hippocampus functionally distinct structures? Neuron 65:7-19 
Godsil BP, Kiss JP, Spedding M, Jay TM (2013) The hippocampalprefrontal pathway: the weak link in psychiatric disorders? Eur Neuropsychopharmacol 23:1165-1181

Hawley DF, Leasure JL (2012) Region-specific response of the hippocampus to chronic unpredictable stress. Hippocampus 22:1338-1349

Hawley DF, Morch K, Christie BR, Leasure JL (2012) Differential response of hippocampal subregions to stress and learning. PLoS ONE 7:e53126

Hessler NA, Shirke AM, Malinow R (1993) The probability of transmitter release at a mammalian central synapse. Nature 366 : $569-572$

Krugers HJ, Alfarez DN, Karst H, Parashkouhi K, van Gemert N, Joëls M (2005) Corticosterone shifts different forms of synaptic potentiation in opposite directions. Hippocampus 15:697-703

Luine V, Villegas M, Martinez C, McEwen BS (1994) Repeated stress causes reversible impairments of spatial memory performance. Brain Res 639:167-170

Luine V, Martinez C, Villegas M, Magariños AM, McEwen BS (1996) Restraint stress reversibly enhances spatial memory performance. Physiol Behav 59:27-32

Maggio N, Segal M (2007) Striking variations in corticosteroid modulation of long-term potentiation along the septotemporal axis of the hippocampus. J Neurosci 27:5757-5765

Maruki K, Izaki Y, Nomura M, Yamauchi T (2001) Differences in paired-pulse facilitation and long-term potentiation between dorsal and ventral CA1 regions in anesthetized rats. Hippocampus 11:655-661

McEwen BS (2012) The ever-changing brain: cellular and molecular mechanisms for the effects of stressful experiences. Dev Neurobiol 72:878-890

McHugh SB, Fillenz M, Lowry JP, Rawlins JN, Bannerman DM (2011) Brain tissue oxygen amperometry in behaving rats demonstrates functional dissociation of dorsal and ventral hippocampus during spatial processing and anxiety. Eur $\mathbf{J}$ Neurosci 33:322-337

Moser MB, Moser EI (1998) Functional differentiation in the hippocampus. Hippocampus 8:608-619

Naber PA, Witter MP (1998) Subicular efferents are organized mostly as parallel projections: a double-labeling, retrograde-tracing study in the rat. J Comp Neurol 393:284-297

Oliveira JF, Dias NS, Correia M, Gama-Pereira F, Sardinha VM, Lima A, Oliveira AF, Jacinto LR, Ferreira DS, Silva AM, Reis JS, Cerqueira JJ, Sousa N (2013) Chronic stress disrupts neural coherence between cortico-limbic structures. Front Neural Circuits 7:1-10
Papatheodoropoulos C, Kostopoulos G (2000a) Decreased ability of rat temporal hippocampal CA1 region to produce long-term potentiation. Neurosci Lett 279:177-180

Papatheodoropoulos C, Kostopoulos G (2000b) Dorsal-ventral differentiation of short-term synaptic plasticity in rat CA1 hippocampal region. Neurosci Lett 286:57-60

Pavlides C, McEwen BS (1999) Effects of mineralocorticoid and glucocorticoid receptors on long-term potentiation in the CA3 hippocampal field. Brain Res 851:204-214

Pêgo JM, Morgado P, Pinto LG, Cerqueira JJ, Almeida OF, Sousa N (2008) Dissociation of the morphological correlates of stressinduced anxiety and fear. Eur J Neurosci 27:1503-1516

Rajah MN, Kromas M, Han JE, Pruessner JC (2010) Group differences in anterior hippocampal volume and in the retrieval of spatial and temporal context memory in healthy young versus older adults. Neuropsychologia 48:4020-4030

Sapolsky RM, Krey LC, McEwen BS (1985) Prolonged glucocorticoid exposure reduces hippocampal neuron number: implications for aging. J Neurosci 5:1222-1227

Segal M, Richter-Levin G, Maggio N (2010) Stress-induced dynamic routing of hippocampal connectivity: a hypothesis. Hippocampus 20:1332-1338

Sotiropoulos I, Cerqueira JJ, Catania C, Takashima A, Sousa N, Almeida OF (2008) Stress and glucocorticoid footprints in the brain-the path from depression to Alzheimer's disease. Neurosci Biobehav Rev 32:1161-1173

Sousa N, Almeida OF (2012) Disconnection and reconnection: the morphological basis of (mal)adaptation to stress. Trends Neurosci 35:742-751

Sousa N, Cerqueira JJ, Almeida OF (2008) Corticosteroid receptors and neuroplasticity. Brain Res Rev 57:561-570

Swanson LW, Wyss JM, Cowan WM (1978) An autoradiographic study of the organization of intrahippocampal association pathways in the rat. J Comp Neurol 181:681-715

Wang XB, Yang Y, Zhou Q (2007) Independent expression of synaptic and morphological plasticity associated with long-term depression. J Neurosci 27:12419-12429

Witter MP, Amaral DG (2004) The hippocampal formation. In: Paxinos $G$ (ed) The rat nervous system. Academic Press, San Diego, pp 635-704

Zucker RS, Regehr WG (2002) Short-term synaptic plasticity. Annu Rev Physiol 64:355-405 